Communications in Physics, Vol.21, No. 2 (2011), pp. 187-191

\title{
GRAVITATIONAL SCALAR FIELD IN 5-DIMENSIONAL KALUZA-KLEIN SPACETIME
}

\author{
NGUYEN THI HA LOAN \\ Hanoi Pedagogical University No. 2
}

\begin{abstract}
In this work the results obtained earlier by the other authors for Gravitational Scalar Fields related to Generally Covariant Duality are extended for the case of Kaluza-Klein Unification model. The equations for these fields are derived explicitly by the use of tetrad postulate and their relation to the scalar field in the metric is considered.
\end{abstract}

\section{INTRODUCTION}

The construction of unification models of all fundamental interactions based on General Relativity in high-dimensional space-time has been a research direction of actual character in particle physics during the last decades. In particular, the problem of extradimensions has been attracted special attention. The pioneer works by Kaluza and Klein [1] have been considered as a meaningful attempt in this direction. On the other hand, in their recent works [2-5] the authors have introduced the conception of covariant Wedge products and generally covariant Duality in the tetrad formalism. In particular some

special scalar fields of gravitational character have been introduced. In this spirit, following the line of unification models based on general relativity with extra-space dimensions, some aspects related to Kaluza-Klein was considered like metrics. The aim of this work is to extend the above mentioned results to the case of Kaluza-Klein Unification model in 5dimentional space-time and to consider the possibility of attaching the gravitational scalar fields to that in Kaluza-Klein metric.

\section{5-DIMENSIONAL LEVI-CIVITA TENSOR}

The Kaluza-Klein metric for the gravitational electromagnetic unification model has been taken in the form

$$
G_{A B}(x)=W(\varphi)\left(\begin{array}{cc}
g_{\mu \nu}(x)+A_{\mu}(x) A_{\nu}(x) \varphi(x) & A_{\mu}(x) \varphi(x) \\
A_{\nu}(x) \varphi(x) & \varphi(x)
\end{array}\right)
$$

where $A_{\mu}(x)$ is electromagnetic field, $\varphi(x)$ is some scalar field, $g_{\mu \nu}(x)$ is 4-dimensional metric, $W(\varphi)$ is some rescaling factor, $A, B$, denote 5- dimensional Lorentz indices, $A$, $B=0,1,2,3,5$ and $\mu, \nu$ denote 4 - dimensional Lorentz indices, $\mu, \nu=0,1,2,3$. Explicitly 
equation (1) means:

$$
\begin{aligned}
& G_{\mu \nu}(x)=W(\varphi)\left(g_{\mu \nu}(x)+A_{\mu}(x) A_{\nu}(x) \varphi(x)\right) \\
& G_{\mu 5}(x)=G_{5 \mu}(x)=W(\varphi) A_{\mu} \varphi(x) \\
& G_{55}(x)=W(\varphi) \varphi(x)
\end{aligned}
$$

These metric components can be expressed in terms of the tetrad satisfying the relation

$$
\begin{aligned}
& G_{A B}(x)=\eta_{M N} q_{A}^{(M)}(x) q_{B}^{(N)}(x) \\
& \eta_{M N}=\operatorname{diag}(1,-1,-1,-1,-1)
\end{aligned}
$$

by putting

$$
\begin{aligned}
& \eta_{a b} q_{\mu}^{(a)} q_{\nu}^{(b)}=W(\varphi) g_{\mu \nu}, \eta_{a b}=\operatorname{diag}(1,-1,-1,-1,-1), a, b=0,1,2,3 \\
& q_{\mu}^{(5)}=i A_{\mu}(W \varphi)^{\frac{1}{2}} \\
& q_{5}^{(a)}=0, q_{5}^{(5)}=i(W \varphi)^{\frac{1}{2}}
\end{aligned}
$$

The corresponding Levi Civita tensors can be constructed as following:

$$
\begin{aligned}
& \varepsilon_{A B C D E}(x)=e_{M N P Q R} q_{A}^{(M)}(x) q_{B}^{(N)}(x) q_{C}^{(P)}(x) q_{D}^{(Q)}(x) q_{E}^{(R)}(x) \\
& \varepsilon^{A B C D E}(x)=e^{M N P Q R} q_{(M)}^{A}(x) q_{(N)}^{B}(x) q_{(P)}^{C}(x) q_{(Q)}^{D}(x) q_{(R)}^{E}(x)
\end{aligned}
$$

where $e_{M N P Q R}$ and $e^{M N P Q R}$ are fully anti-symmetric tensors with $\varepsilon_{01235}=\varepsilon^{01235}=+1$. We now put

$$
\begin{aligned}
& \varepsilon^{A B C D E}(x)=e^{A B C D E} \cdot B(x) \\
& \varepsilon_{A B C D E}(x)=e_{A B C D E} \cdot C(x)
\end{aligned}
$$

where $B(x)$ and $C(x)$ are some one-component fields transforming according to the law

$$
B^{\prime}\left(x^{\prime}\right)=J B(x), \quad C^{\prime}\left(x^{\prime}\right)=J^{-1} C(x)
$$

under general transformation

$$
x^{A} \rightarrow x^{A}=f^{A}(x)
$$

where $J$ stands for the determinant of the $5 \times 5$ matrix with $\frac{\partial x^{\prime A}}{\partial x^{B}}$ as element on row $A$ and column $B$,

$$
J \equiv D\left(\frac{x^{\prime}}{x}\right) \equiv\left|\begin{array}{lllll}
\frac{\partial x^{\prime 0}}{\partial x^{0}} & \frac{\partial x^{\prime 0}}{\partial x^{1}} & \frac{\partial x^{\prime 0}}{\partial x^{2}} & \frac{\partial x^{\prime 0}}{\partial x^{3}} & \frac{\partial x^{\prime 0}}{\partial x^{5}} \\
\frac{\partial x^{\prime 1}}{\partial x^{0}} & \frac{\partial x^{\prime 1}}{\partial x^{1}} & \frac{\partial x^{\prime 1}}{\partial x^{2}} & \frac{\partial x^{\prime 1}}{\partial x^{3}} & \frac{\partial x^{\prime 1}}{\partial x^{5}} \\
\frac{\partial x^{\prime 2}}{\partial x^{0}} & \frac{\partial x^{\prime 2}}{\partial x^{1}} & \frac{\partial x^{\prime 2}}{\partial x^{2}} & \frac{\partial x^{\prime 2}}{\partial x^{3}} & \frac{\partial x^{\prime 2}}{\partial x^{5}} \\
\frac{\partial x^{\prime 3}}{\partial x^{0}} & \frac{\partial x^{\prime 3}}{\partial x^{1}} & \frac{\partial x^{\prime 3}}{\partial x^{2}} & \frac{\partial x^{\prime 3}}{\partial x^{3}} & \frac{\partial x^{\prime 3}}{\partial x^{5}} \\
\frac{\partial x^{\prime 5}}{\partial x^{0}} & \frac{\partial x^{\prime 5}}{\partial x^{1}} & \frac{\partial x^{\prime 5}}{\partial x^{2}} & \frac{\partial x^{\prime 5}}{\partial x^{3}} & \frac{\partial x^{\prime 5}}{\partial x^{5}}
\end{array}\right|
$$


The formula (7) shows that $B(x)$ and $C(x)$ are scalar fields with respect only to transformations with $J=1$ but not to general transformation. Note also that the field defined as

$$
F(x) \equiv B(x) \cdot C(x)=\frac{1}{5 !} \varepsilon^{A B C D E}(x) \varepsilon_{A B C D E}(x)
$$

is scalar with respect to general transformation. The inverse formulae of (6) are:

$$
\begin{aligned}
& B(x)=\frac{1}{5 !} e_{A B C D E} \varepsilon^{A B C D E}(x) \\
& C(x)=\frac{1}{5 !} e^{A B C D E} \varepsilon_{A B C D E}(x)
\end{aligned}
$$

The possibility of using this field as $\varphi(x)$ in Kaluza-Klein metric (1) will be discussed in the last section.

\section{BASIC EQUATIONS}

In this section we derive the basic equations for $B(x)$ and $C(x)$ defined by the formulae (6), following the method analogous to that of Ref. [5].

From the tetrad postulate for covariant derivative

$$
D_{B} q_{A}^{(M)}(x)=0
$$

and the tetrad structure (5), it follows immediately:

$$
D_{F} \varepsilon^{A B C D E}(x)=0, \quad D_{F} \varepsilon_{A B C D E}(x)=0
$$

The equations (11) lead to the following equations for $B(x)$ and $C(x)$ defined by (9):

$$
\begin{aligned}
& \left(\partial_{A}+\Gamma_{A D}^{D}\right) B(x)=0 \\
& \left(\partial_{A}-\Gamma_{A D}^{D}\right) C(x)=0
\end{aligned}
$$

and therefore

$$
\begin{aligned}
& \left\{\square^{(5)}-\eta^{A B} \Gamma_{A C}^{C} \Gamma_{B D}^{D}+\partial^{A} \Gamma_{A C}^{C}\right\} B(x)=0 \\
& \left\{\square^{(5)}-\eta^{A B} \Gamma_{A C}^{C} \Gamma_{B D}^{D}-\partial^{A} \Gamma_{A C}^{C}\right\} C(x)=0
\end{aligned}
$$

where $\Gamma_{A C}^{C}$ is 5 - dimensional affine connection,

$$
\Gamma_{B C}^{A}=\frac{1}{2} G^{A D}\left(\partial_{B} G_{D C}+\partial_{C} G_{D B}-\partial_{D} G_{B C}\right)
$$

$\square^{(5)} \equiv \partial^{A} \partial_{A}$ is 5 - dimensional Dalembert operator.

To calculate the quantities entered the equation (12) we will use the well known formula

$$
\Gamma_{A C}^{C}=\frac{1}{2 G} \partial_{A} G
$$

where $G$ denotes the determinant of the metric tensor $G_{A B}$, which for the case (1) equals

$$
\begin{aligned}
& G=W^{5}(\varphi) \varphi g \\
& g \equiv \operatorname{det}\left(g_{\mu \nu}\right)
\end{aligned}
$$


The 4- dimensional metric $g_{\mu \nu}$ is traditionally decomposed as

$$
g_{\mu \nu}(x)=\eta_{\mu \nu}+h_{\mu \nu}(x)
$$

where $h_{\mu \nu}(x)$ is referred to as gravitational field, and hence, $g \approx-\left(1+h_{\mu}^{\mu}\right)$ up to first order in $h_{\mu \nu}$.

Furthermore, let us restrict ourselves to effective theory approximation, where the metric tensor is considered not to depend on the extra coordinate $x^{5}$. In this case, the calculations give (up to first order in $h_{\mu \nu}(x)$ ):

$$
\begin{aligned}
& \eta^{A B} \Gamma_{A C}^{C} \Gamma_{B D}^{D}=\frac{1}{4\left(W^{5} \varphi\right)^{2}} \partial^{\mu}\left(W^{5} \varphi\right)\left\{\partial_{\mu}\left(W^{5} \varphi\right)+2\left(W^{5} \varphi\right) \partial_{\mu} h\right\} \\
& \partial^{A} \Gamma_{A C}^{C}=\frac{1}{2} \partial^{\mu}\left\{\frac{1}{W^{5} \varphi} \partial_{\mu}\left(W^{5} \varphi\right)+\partial_{\mu} h\right\} \\
& h \equiv \eta^{\mu \nu} h_{\mu \nu}
\end{aligned}
$$

Let us consider two following special cases of the rescaling factor $W(\varphi)$

1) $W(\varphi)=1$

Equations (12) and (16) then give:

$$
\begin{aligned}
& \left\{\square-\frac{1}{4 \varphi^{2}} \partial^{\mu} \varphi\left(\partial_{\mu} \varphi+2 \varphi \partial_{\mu} h\right)+\frac{1}{2} \square(\ln \varphi+h)\right\} B(x)=0 \\
& \left\{\square-\frac{1}{4 \varphi^{2}} \partial^{\mu} \varphi\left(\partial_{\mu} \varphi+2 \varphi \partial_{\mu} h\right)-\frac{1}{2} \square(\ln \varphi+h)\right\} C(x)=0
\end{aligned}
$$

2) $W(\varphi) \sim \varphi^{-5}$

Equations (12) and (16) then give:

$$
\begin{aligned}
& \left(\square+\frac{1}{2} \square h\right) B(x)=0 \\
& \left(\square-\frac{1}{2} \square h\right) C(x)=0
\end{aligned}
$$

These equations have the similar form as those for 4- dimensional space-time obtained in Ref. [5].

\section{METRIC SCALAR FIELD}

In this section we consider the possibility of using the gravitational scalar field defined by (8) as the scalar field appeared in Kaluza-Klein metric (1). For definiteness let us put the rescaling factor to be $W(\varphi)=1$. We start from the metric compatibility condition

$$
D_{A} G_{B C}=\partial_{A} G_{B C}-\Gamma_{A B}^{D} G_{D C}-\Gamma_{A C}^{D} G_{B D}=0
$$

For the component $G_{55}$ this implies:

$$
\begin{aligned}
& \partial_{5} G_{55}-2\left(\Gamma_{55}^{5} G_{55}+\Gamma_{55}^{\mu} G_{\mu 5}\right)=0 \\
& \partial_{\mu} G_{55}-2\left(\Gamma_{\mu 5}^{5} G_{55}+\Gamma_{\mu 5}^{\nu} G_{\nu 5}\right)=0
\end{aligned}
$$


For the affine connections the calculations give:

$$
\begin{aligned}
\Gamma_{55}^{5} & =\frac{1}{2}\left(\varphi^{-1}+A . A\right) \partial_{5} \varphi-\frac{1}{2} A^{\nu}\left[2 \partial_{5}\left(A_{\nu} \varphi\right)-\partial_{\nu} \varphi\right] \\
\Gamma_{55}^{\mu} & =-\frac{1}{2} A^{\mu} \partial_{5} \varphi+\frac{1}{2} g^{\mu \nu}\left[2 \partial_{5}\left(A_{\nu} \varphi\right)-\partial_{\nu} \varphi\right] \\
\Gamma_{\mu 5}^{5} & =\frac{1}{2}\left(\varphi^{-1}+A . A\right) \partial_{\mu} \varphi-\frac{1}{2} A^{\nu}\left[\partial_{\mu}\left(A_{\nu} \varphi\right)-\partial_{\nu}\left(A_{\mu} \varphi\right)+\partial_{5}\left(g_{\mu \nu}+A_{\mu} A_{\nu} \varphi\right)\right] \\
\Gamma_{\mu 5}^{\nu} & =-\frac{1}{2} A^{\nu} \partial_{\mu} \varphi+\frac{1}{2} g^{\nu \rho}\left[\partial_{\mu}\left(A_{\rho} \varphi\right)-\partial_{\rho}\left(A_{\mu} \varphi\right)+\partial_{5} g_{\rho \mu}\right]
\end{aligned}
$$

where $A . A \equiv A^{\mu} A_{\mu} \equiv g^{\mu \nu} A_{\mu} A_{\nu}$.

With the expressions (22) the condition (20) is satisfied identically, while the condition (21) becomes:

$$
A^{\nu} \varphi \cdot \partial_{\mu}\left(A_{\mu} A_{\nu} \varphi\right)=0
$$

or

$$
(A . A) \cdot A_{\mu} \varphi \cdot \partial_{5} \varphi=0
$$

when the electromagnetic field $A_{\mu}$ does not depend on $x^{5}$.

The condition (24) will be satisfied for any $\varphi$ not depending on $x^{5}$. In particular it would be the component $F^{(0)}$ in the Fourier expansion of $F(x)$ :

$$
F(x)=\sum_{n=-\infty}^{\infty} F^{(n)}\left(x^{\mu}\right) e^{i n \frac{x^{5}}{R_{5}}} \quad, \quad 0 \leq x^{5} \leq 2 \pi R_{5}
$$

where $R_{5}$ is the radius of compactification circle for extradimension $x^{5}$.

\section{CONCLUSION}

In this work we have considered some aspects of Kaluza-Klein metric related to the unification model with higher dimensional space-time. The main result obtained is that concerning the scalar field appearing in the metric, showing that this field would be of originally gravitational character. In this paper only preliminary results were found, the physical meaning of the scalar field will be clarified in next study.

\section{REFERENCES}

[1] H. C. Lee, An introduction to Kaluza- Klein theories, World Scientific, 1983.

[2] D. N. Diep, D. V. Duc, H. V. Tan, N. A. Viet, Convolution Wedge Product of Fields, ICTP preprint, IC/2007/056, Trieste, 2007.

[3] D. N. Diep, D. V. Duc, H. V. Tan, N. A. Viet, Comm. in Physics 19 (2007) 99.

[4] D. N. Diep, D. V. Duc, H. V. Tan, N. A. Viet, Proceeding of the $33^{\text {rd }}$ National Conference on Theoretical Physics, Da Nang, 2008.

[5] Dao Vong Duc, A Model for String Field Interaction Lagrangian, Report at the $35^{\text {th }}$ National Conference on Theoretical Physics, Ho Chi Minh City 2010.

Received 29 October 2010. 